\title{
QUALITY IMPROVEMENT Improving DVLA advice provided to the patients with acute coronary syndrome upon discharge
}

\author{
Authors: Tamara Naneishvili, ${ }^{A}$ Arsalan Khalil, ${ }^{B}$ Abigail Mayo-Evans ${ }^{C}$ and James Glancy ${ }^{D}$
}

Acute coronary syndrome (ACS) and coronary intervention can significantly impair patients' ability to drive and, therefore, the Driver \& Vehicle Licensing Agency (DVLA) provides relevant guidance for patients and healthcare professionals on driving in order to safeguard patients, passengers and public in general.

The initial pre-teaching cohort analysis revealed that $12.9 \%$ of the discharge summaries had documented driving advice and only $3.23 \%$ were in accordance with the DVLA guidance. Our primary aim was to increase the provision of appropriate driving advice to $>90 \%$. Secondary aims were to improve the quality of the written advice and to increase junior doctors' awareness and confidence in doing so.

We created a template with standardised driving advice with specific guidance for group 1 and group 2 drivers, delivered formal and informal teaching, and distributed information leaflets. These measures led to an overall improvement in provision of correct advice to $>90 \%$.

We demonstrated how simple measures of introducing a standardised driving advice template and conducting formal and informal teaching could significantly improve the quality of current practice pertaining to the DVLA's driving restrictions in ACS patients. The successful strategies employed by us can be utilised by other trusts across the UK to promote person-centred care and improve patient safety.

KEYWORDS: acute coronary syndrome, DVLA, quality improvement project, cardiology

DOI: $10.7861 /$ fhj.2020-0196

\section{Introduction}

Hereford County Hospital caters for the majority of the patients presenting with acute coronary syndrome (ACS) in the Herefordshire region. We noted that written guidance on driving restrictions in patients with ACS was seldom provided on discharge summaries and the quality of the documented advice was variable. Baseline measurements showed that $13 \%$ (4/31) of all discharge summaries

Authors: A cardiology registrar, Wye Valley NHS Trust, Hereford, UK; ${ }^{B}$ internal medicine trainee, Wye Valley NHS Trust, Hereford, UK; ${ }^{C}$ physician associate, Wye Valley NHS Trust, Hereford, UK;

${ }^{D}$ cardiology consultant, Wye Valley NHS Trust, Hereford, UK for patients having ACS included driving advice and only 3\% (1/31) were correct.

Many cardiac conditions and interventions can affect driving ability and, as a result, require temporary driving restrictions. ${ }^{1}$ Informing patients of these restrictions is a professional and legal requirement. ${ }^{2}$ Discussions should be held with the patient and also documented in the discharge summary. Lack of documentation can have serious implications for the safety of patients and the general public.

The primary aim was to increase the provision of driving advice to these patients upon discharge from $13 \%$ to $>90 \%$. The secondary aim was to improve the quality of the written advice and to increase junior doctors' awareness.

\section{Methods and measurements}

Baseline data were collected from 01 June 2019 to 31 August 2019 in the cardiology ward at Hereford County Hospital. All patients with ACS were identified from the cardiology inpatient Myocardial Ischaemia National Audit Project (MINAP). Electronic discharge summaries were assessed for data collection.

\section{Intervention 1}

To increase junior doctors' awareness of the Driver \& Vehicle Licensing Agency (DVLA) guidance in patients with ACS, we created a template with standardised driving advice, which was made available on the cardiology ward. Printed copies were also distributed. Data were collected in October 2019.

\section{Intervention 2}

Informal teaching was undertaken and to make the templates readily available, electronic versions were sent via email. Data were gathered in November 2019.

\section{Intervention 3}

Junior doctors rotate every 4 months, therefore, hardcopies of the DVLA guidance was included in the junior doctor's induction programme. Data were collected in December 2019.

\section{Intervention 4}

Further informal teaching pertaining to DVLA guidance during morning medical handovers was delivered. Final data were collected in January 2020. 


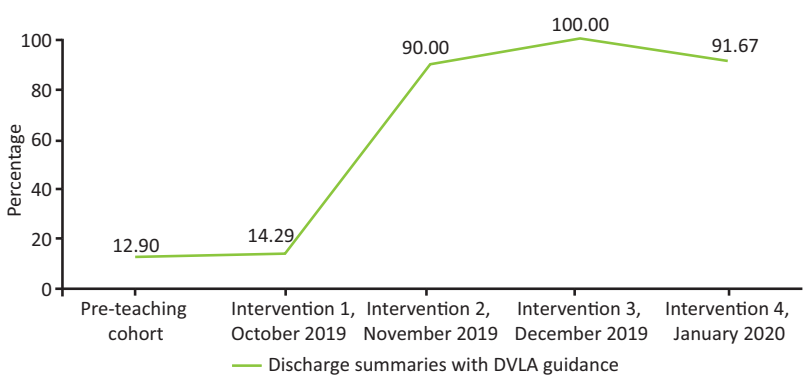

Fig 1. Percentage of discharge summaries with Driver \& Vehicle Licensing Agency guidance. DVLA = Driver \& Vehicle Licensing Agency.

\section{Results}

The total number of discharge summaries in the pre-teaching cohort was 31 and varied from seven to 12 for the subsequent cycles.

The initial pre-teaching cohort analysis revealed that 13\% (4/31) of the discharge summaries had documented driving advice and only 3\% (1/31) were in accordance with the DVLA guidance. After the introduction of the standardised DVLA guidance template and distribution of hard copies, we saw a minimal improvement to $14 \%(1 / 7)$.

Subsequently, we decided to conduct informal teaching and distributed electronic versions of DVLA templates. Adoption of electronic copies of DVLA templates led to a massive rise from a previous value of $14 \%$ (1/7) to $90 \%$ (9/10; Fig 1), all of which were in compliance with the DVLA guidance (Box 1).

\section{Box 1. Driver \& Vehicle Licensing Agency guidance for acute coronary syndrome, March 2021}

\section{ACS with no intervention: \\ Group 1 licence: 4 weeks of no driving and no need to inform DVLA if there are no other disqualifying conditions Group 2 licence: no driving and must notify DVLA.}

\section{ACS with successful PCI and EF $>40 \%$ with no plan for revascularisation in 4 weeks:}

Group 1 licence: can resume driving in 1 week and no need to inform DVLA if there are no other disqualifying conditions Group 2 licence: must not drive and must inform DVLA; licence may be reissued in 6 weeks if functional tests are met.

\section{Elective PCI}

Group 1 licence: can resume driving in 1 week and no need to inform DVLA if there are no other disqualifying conditions Group 2 licence: must not drive and must inform DVLA; licence may be reissued in 6 weeks if functional tests are met.

ACS = acute coronary syndrome; DVLA = Driver \& Vehicle Licensing Agency; $\mathrm{EF}=$ ejection fraction; $\mathrm{PCI}=$ percutaneous coronary intervention.
Following changeover, the new batch of junior doctors was given formal teaching and information leaflets during their induction. This was a very effective measure which led to a $100 \%(12 / 12)$ documentation of appropriate DVLA guidance on discharge summaries.

Inclusion of guidance regarding DVLA regulation in morning handovers helped to maintain the compliance above 90\% (9/10). All of this was in accordance with the DVLA guidance (10/10).

\section{Discussion}

ACS with or without percutaneous coronary intervention can temporarily impair patients' ability to drive. Therefore, it is very important for doctors to inform their patients of these restrictions.

We demonstrated that simple educational interventions and a standardised DVLA template improved the quality and quantity of provided driving advice. Incorporating the template into junior doctors' inductions ensured the sustainability of these practices.

Moreover, providing the driving advice on discharge from hospital is not limited to ACS and similar interventions may be beneficial in other cardiac conditions as well. Therefore, we would encourage junior doctors elsewhere to identify similar problems in their clinical areas and come up with quality improvement interventions to improve patient safety.

We acknowledge that the number of patients in our project is a limitation. Projects comprising a larger cohort of patients in tertiary care centres would be more helpful.

\section{Conclusion}

The combination of a template for standardised advice with education and inclusion in induction is required to achieve reliable advice to patients and general practitioners. This can be applied in many other units, and with other safety advice. .

\section{References}

1 Driver and Vehicle Licensing Agency. Assessing fitness to drive: a guide for medical professionals. DVLA, 2021.

2 General Medical Council. Good medical practice. GMC, 2013.

Address for correspondence: Dr Tamara Naneishvili, Hereford County Hospital, Stonebow Road, Hereford HR1 2BN, UK. Email: tamara.naneishvili@nhs.net 